\title{
Leclercia adecarboxylata Infection in Protein Energy Malnutrition (PEM) Patient: A Case Report
}

\author{
Vijayshri Deotale ${ }^{1}$, Yogendra Shelke ${ }^{2}$, Deepashri Maraskolhe ${ }^{3}$, Ruchita Attal ${ }^{4}$ \\ ${ }^{1,2,3,4}$ Department of Microbiology, Mahatma Gandhi institute of Medical science (MGIMS) Sevagram, Wardha, State - Maharashtra India
}

\begin{abstract}
Normal conmmensal commonly leads to complications in immunocopramised patients which leads to increased mortality and morbidty. Laclercia adecarboxylata is an unusual emerging pathogen in healthy and imunocompranised patients. Here, we report a first case report of Leclercia adecarboxylata in a pediatric patient with a lower respiratory tract infection associated with PEM grade III.
\end{abstract}

Keywords: Leclercia adecarboxylata, immunocompramised paediatric infection

\section{Introduction}

Leclercia adecarboxylata is an ubiquitous bacterium of the Enterobacteriaceae family, has been rarely isolated from environmental and clinical specimens. It was first described by Leclerc in 1962 as Escherichia adecarboxylata [0], but additional biochemical assessments showed that it was a distinct genus, therefore it was renamed Leclercia adecarboxylata in honor of Leclerc [[2]]. It is a aerobic and facultative anaerobic, Gram negative, oxidase negative, mesophilic bacteria which is motile by peritrichus flagella. Commonly isolated from patients with bacteremia, peritonitis, gallbladder infection, neutropenia. Most human infections are polymicrobial [[3]]. Only few clinical case reports are published and most of the clinical reports are from immunocompramised host [[4]]. It is rarely pathogenic in immunocompetent patient [[5]]. The organism is invariably sensitive to most antibiotics but there are reports of resistant strains also [[6]],[[7]].

\section{Case Report}

Two-year old girl presented with complain of high grade intermittent fever which was not associated with chills and rigor, common cold with nonproductive cough and vomiting. On anthropological examination, showed weight $7.8 \mathrm{~kg}$, head circumference $43 \mathrm{~cm}$, length $71 \mathrm{~cm}$, indicating PEM grade III. So patient was investigated for Complete blood count, blood culture, reticulocyte count \& $\mathrm{Hb}$ electrophoresis. Complete blood count (CBC) showed total

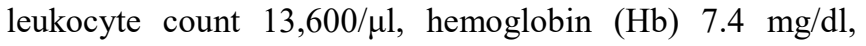
peripheral smear showed microcytic hypochromic RBC indicating iron deficiency anemia. Red cell distribution width (RDW) was increased and showed bipenia with microcytic hypochromic anemia on peripheral smear. Creactive protein (CRP) was negative. Patient was positive for sickling test. Conventional blood culture showed pure growth of single organism on first day of incubation. Colony morphology on Blood agar was non-haemolytic, large bisected colonies with yellow pigmentation (Fig-1). Since the sample was blood culture for isolation of Haemophillus a Staphylococcus streaking was done on the primary and secondary culture streaking. Growth on MacConkey agar showed lactose fermenting mucoid colonies. Gram staining of colony showed Gram negative bacilli approximately 1-
$3 \mu \mathrm{m}$, which were motile, catalase positive and oxidase negative. By conventional biochemical method glucose, lactose, mannitol and sucrose were utilized with production of acid with out gas, malonate utilization (positive) Indole and Methyl red test positive, nitrate was reduced to nitrite, but negative for lysine and ornithine decarboxylase, arginine dihydrolase. According to conventional method organisms was placed into family Enterobacteriaceae and tribe Escherichiae. The results for susceptibility testing were interpreted according to Clinical and Laboratory Standards Institute CLSI guideline 2015.

Organism was also subjected to Vitek 2 (BioMerieux) automated identification and sensitivity testing. An organism was named as Leclercia adecarboxylata which was susceptible to all subjected antibiotics.

Patient was given oral amoxicillin, but it induced diarrhea in child therefore shifted to oral cefixime (syrup) and patient responded to cefixime very well and discharged on $3^{\text {rd }}$ day.

\section{Discussion}

This is the first case report from Central India as pure growth was isolated from blood culture with 24 hour of incubation of pediatric patient. There were few reported cases isolated from blood culture of pediatric age group listed in Table -1, but all of them were from outside India. The epidemiological significance of L.adecarboxylata is not clear. The paucity of reports of human infection may reflect misdiagnosis, as the organisms share many biochemical features with E.coli rather than true infrequency of human infection.

Only few cases has been reported in pediatrics age groups. Most of them are associated with infants or preterm babies and all of them has underling severe disease (Table 1).

Our patient was having Protein Energy Malnutrition (PEM) $\&$ iron deficiency anemia, sickling test was positive. It is interesting to find correlation between PEM, anemia as predisposing factor for mono infection of L.adecarboxylata.

On blood agar plate the colonies were very typical bisected (Fig-1). At that juncture it appeared to be some unusual isolate. Hence, we could capture the image of culture plate. But on subsequent subculture we could not reproduce same 


\section{International Journal of Science and Research (IJSR) \\ ISSN (Online): 2319-7064 \\ Index Copernicus Value (2013): 6.14 | Impact Factor (2014): 5.611}

type of colonies. This could be because of prolonged enrichment of sample in Brain Heart infusion. In our case we have identified the organism by using extended biochemical reactions even this large group of phenotypic test; one cannot be assured that identification is $100 \%$ accurate. Nucleic acid analysis especially 16s Ribosomal DNA should be more accurate but due to unavailability of this test in our set up, we process growth in Vitek 2 system by using Gram Negative card for accurate identification.

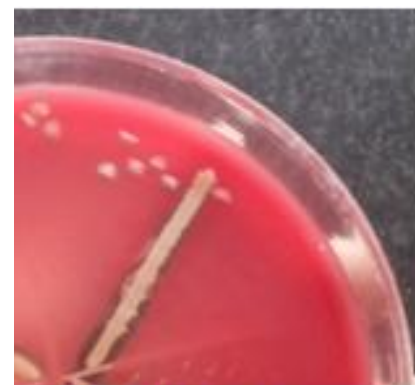

Figure 1-"a"

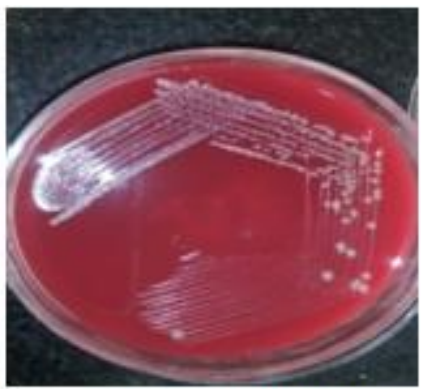

Figure 1- "b"
Figure 1: ("a" and "b"): Growth on 5\% sheep Blood Agar showing bisected colonies

There are scattered reports of such cases occurring in immunocompetent patients including a positive blood culture in an asymptomatic platelet donor [[8]]

It would be speculative to conclude how our patient came in contact with the bacterium. Our patient's clinical course was relatively mild, as compared to all reported cases Most of them associated with severe underling disease, immunodeficiency in terms of organ transplant, chronic disease or chemotherapy but in our case there is no underlying diagnosed major illness till date.

Table 1: Association of isolation of Laclercia adecarboxylata with immune status in paediatric age group from blood culture

\begin{tabular}{|c|c|c|c|c|c|}
\hline Sr. No & $\begin{array}{l}\text { Culture } \\
\text { source }\end{array}$ & $\begin{array}{c}\text { Associated bacterial/ } \\
\text { fungal co-infection }\end{array}$ & Age/sex & $\begin{array}{c}\text { Underlying } \\
\text { immunodeficiency }\end{array}$ & References \\
\hline 1. & Blood & None & $8 \mathrm{mth} \mathrm{M}$ & $\begin{array}{l}\text { Intestinal atresia, } \\
\text { patient on parental } \\
\text { nutrition }\end{array}$ & \begin{tabular}{|c|} 
Otani E, Bruckner DA (1991) Leclercia adecarboxylata \\
isolated from blood culture.Clin Microbiol Newslett 13:157- \\
158.
\end{tabular} \\
\hline 2. & Blood & None & $26 w k F$ & $\begin{array}{c}\text { Premature,respiratory } \\
\text { distress,sepsis }\end{array}$ & $\begin{array}{l}\text { Myers KA, Jeffery RM, Lodha A (2012) Late-onset } \\
\text { Leclercia adecarboxylata } \\
\text { bacteraemia in a premature infant in the NICU. Acta } \\
\text { Paediatr 101: e37-39. }\end{array}$ \\
\hline 3. & Blood & Staphylococcus aureus & $11 \mathrm{mth} F$ & Leukemia & $\begin{array}{l}\text { Longhurst CA, West DC (2001) Isolation of Leclercia } \\
\text { adecarboxylata from an } \\
\text { infant with acute lymphoblastic leukemia. Clin Infect Dis } \\
\text { 32: } 1659 .\end{array}$ \\
\hline 4. & Blood & None & $5 \mathrm{M}$ & Sepsis & $\begin{array}{l}\text { Sethi K, Barker EM, Metlay LA, Caserta MT, Daugherty } \\
\text { LE. Leclercia adecarboxylata Sepsis and Cerebral } \\
\text { Herniation. J Pediatr Infect Dis Soc . 2014;3 (1 ):e1-e3. }\end{array}$ \\
\hline 5. & Blood & None & 24 wk F & $\begin{array}{c}\text { Sepsis in premature } \\
\text { infant }\end{array}$ & $\begin{array}{l}\text { Nelson MU, Maksimova Y, Schulz V, Bizzarro MJ, } \\
\text { Gallagher PG. Late-onset Leclercia adecarboxylata sepsis in } \\
\text { a premature neonate. J Perinatol. 2013;33(9):740-742. }\end{array}$ \\
\hline 6. & Blood & & $\begin{array}{c}16 \text { days } \\
\text { old }\end{array}$ & $\begin{array}{c}\text { Sepsis in premature } \\
\text { infant }\end{array}$ & $\begin{array}{c}\text { Myers, K., Jeffery, R. and Lodha, A. (2012),Late-onset } \\
\text { Leclercia adecarboxylata bacteraemia in a premature infant } \\
\text { in the NICU. Acta Paediatrica, 101: e37-e39. }\end{array}$ \\
\hline 7. & Blood & None & 5 yr old & $\begin{array}{c}\text { End-stage renal } \\
\text { disease, peritonitis }\end{array}$ & $\begin{array}{c}\text { Fattal O, Deville JG. Leclercia adecarboxylata peritonitis in } \\
\text { a child receiving chronic peritoneal dialysis. Pediatr } \\
\text { Nephrol. } 2000 ; 15(3-4): 186-187 .\end{array}$ \\
\hline
\end{tabular}

\section{References}

[1] Leclerc H, "Etude biochimique d'enterobacteriaceae pigmente'es,". Ann.Inst. Pasteur, CII, pp. 726-741, 1962.

[2] Tamura K, Sakazaki R, Kosako Y, Yoshizaki E, “ Leclercia adecarboxylata gen. nov., comb. nov., formerly known as Escherichia adecarboxylata," Curr Microbiol, XIII (4), pp. 179-184, 1986.

[3] Prakash MR, Ravikumar R, Patra N, Indiradevi B, "Hospital acquired pneumonia due to Leclercia adecarboxylata in a neurosurgical centre, " J.Postgrad Med ,LXI (2), pp.123-125, 2015.

[4] Benjamin H, Andrew B, and Mark K.H, "Leclercia adecarboxylata in a $\mathrm{n}$ immunocompetent patient," J Med Microbiol LVII,pp. 896-989, 2008.
[5] Edward H. H, Eric C, Julia A. K, Richard K, "Leclercia Adecarboxylata infection in an Immunocompetent Child," RIMJ, pp. 41-44, 2015.

[6] Stock I , Burak S and Wiedemann B, "Natural antimicrobial susceptibility pattern and biochemical profiles of Lecrecia adecarboxylata strains," Clin. Microbiol Infect, X, pp.724-733,2004.

[7] Temesgen Z, Toal DR, Cockerill FR, "Leclercia adecarboxylata infections: case report and review," Clin Infect Dis XXV, pp. 79-81, 1997.

[8] Davenport P, Land KJ, "Isolation of Leclercia adecarboxylata from the blood culture of an asymptomatic platelet donor," Transfusion, XLVII (10), pp.1816-1819, 2007. 